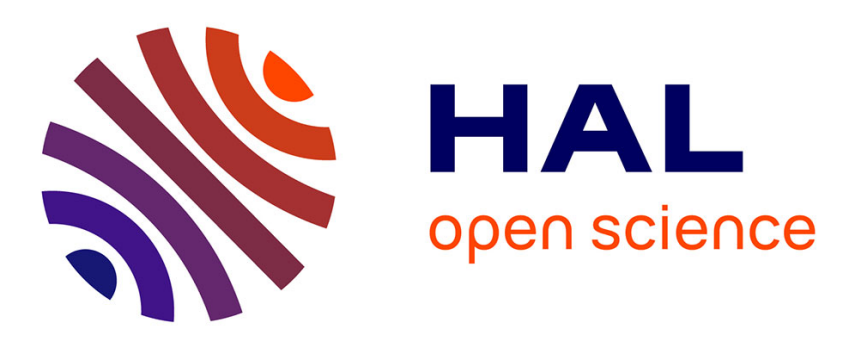

\title{
Analysis of Parameter Variability in an Integrated Wireless Power Transfer System via Partial Least Squares Regression
}

Mourad Larbi, Riccardo Trinchero, Flavio Canavero, Philippe Besnier, Madhavan Swaminathan

\section{To cite this version:}

Mourad Larbi, Riccardo Trinchero, Flavio Canavero, Philippe Besnier, Madhavan Swaminathan. Analysis of Parameter Variability in an Integrated Wireless Power Transfer System via Partial Least Squares Regression. IEEE Transactions on Components, Packaging and Manufacturing Technology, 2020, 10 (11), pp.1795-1802. 10.1109/TCPMT.2020.3002226 . hal-02905733

\section{HAL Id: hal-02905733 \\ https://hal.science/hal-02905733}

Submitted on 23 Jul 2020

HAL is a multi-disciplinary open access archive for the deposit and dissemination of scientific research documents, whether they are published or not. The documents may come from teaching and research institutions in France or abroad, or from public or private research centers.
L'archive ouverte pluridisciplinaire HAL, est destinée au dépôt et à la diffusion de documents scientifiques de niveau recherche, publiés ou non, émanant des établissements d'enseignement et de recherche français ou étrangers, des laboratoires publics ou privés. 


\title{
Analysis of Parameter Variability in an Integrated Wireless Power Transfer System via Partial Least Squares Regression
}

\author{
Mourad Larbi, Riccardo Trinchero, Member, IEEE, Flavio G. Canavero, Fellow, IEEE, Philippe Besnier, Senior \\ Member, IEEE, Madhavan Swaminathan, Fellow, IEEE
}

\begin{abstract}
This paper deals with the application of the partial least squares (PLS) regression to the uncertainty quantification of an integrated wireless power transfer with 30 random variables. It considers the development of surrogate models using a limited set of training samples in order to estimate statistical quantities of the converter efficiency with a relatively low computational cost compared to the standard brute force Monte Carlo (MC) simulation. The strength, the performance and the features of the proposed modeling approach are then compared with the ones of an advanced probabilistic surrogate model combining the Leastsquares Support Vector Machine (LS-SVM) and the Gaussian Process (GP) regression. The MC simulation is considered as reference.
\end{abstract}

Index Terms-Machine learning, uncertainty quantification, surrogate model, Gaussian Process regression, Least-squares Support Vector Machine regression, PLS regression, sensitivity analysis, probabilistic model, wireless power transfer (WPT).

\section{INTRODUCTION}

The level of complexity of electronic systems is so high nowadays that it becomes a difficult task to guarantee their reliability. Due to the fabrication process, the variability of parameters associated with the system has to be considered, since it may generate large variations of output signals and lead to a potential failure of systems. In the above scenario, uncertainty quantification (UQ) plays a key role since it allows predicting the effect of the uncertain parameters on the system performance. In advanced electronic systems, the complexity and the simulation costs are so high that the brute force Monte Carlo (MC) approach turns out to be inefficient.

Alternatively, various surrogate models have been successfully proposed. One of the most used techniques is the polynomial chaos (PC), which has shown good performance to solve different applications [1]-[3]. Despite its accuracy and its improved efficiency with respect to the plain MC simulation

This work has been supported by the Joint Project for Internalization of Research of Politecnico di Torino (2018): "Machine Learning to Improve the Reliability of Complex Systems" and 3D Systems Packaging Research Center, Georgia Tech.

Mourad Larbi and Madhavan Swaminathan are with the School of Electrical and Computer Engineering, Georgia Institute of Technology, Atlanta, GA 30332 (e-mail: mourad.larbi@ece.gatech.edu, madhavan.swaminathan@ece.gatech.edu).

Riccardo Trinchero and Flavio G. Canavero are with the Department of Electronics and Telecommunications, Politecnico di Torino, Torino 10129 , Italy (e-mail: riccardo.trinchero@polito.it, flavio.canavero@polito.it).

Philippe Besnier is with the IETR, UMR CNRS 6164: Institut d'Electronique et de Télécommunications de Rennes, INSA de Rennes, 35708 Rennes, France (e-mail: philippe.besnier@insa-rennes.fr). in terms of simulation costs, such technique suffers the socalled curse of dimensionality, since the number of unknown coefficients to be estimated during the training of the PCsurrogate grows exponentially with respect to the number of uncertain parameters and the expansion order. To this aim several advanced implementations of the PC expansion such as tensor and sparse formulations have been presented to bypass the mentioned problem [4]-[7].

Recently, alternative solutions based on Machine Learning (ML) regressions have been investigated in order to mitigate the above issues. In particular, non-parametric regressions (i.e., techniques in which the regression complexity is independent from the dimensionality of the parameter space) such as support vector machine (SVM) and least-squares support vector machine (LS-SVM) regression [8]-[11], Gaussian process regression (GPR) [12]-[14] and their combinations (e.g., the LS-SVM+GPR surrogate model) [15]-[17] can be considered as valuable alternative techniques to the state-ofthe-art approaches for the UQ in complex systems.

This paper presents an alternative technique for the UQ in high-dimensional problems [18]-[20], such as the partial least squares (PLS) regression [21]. The PLS regression allows to build a surrogate model of an output depending on many uncertain parameters. Similarly to the principal component analysis (PCA) [22], PLS allows to reduce the problem dimensionality using a limited number of components while mapping the relationship between input and output variables. This property of the method is very interesting as it captures the most prominent input variables of the systems. Moreover, the surrogate model can then be used to compute statistical moments and the probability density function (PDF) of the output of interest. The accuracy and the strength of the proposed modeling scheme are then investigated by comparing the results of the surrogate model built via the PLS and the ones provided by a probabilistic model based on the LSSVM+GPR based on the results of a MC simulations. The above techniques are applied for the UQ of the efficiency of a wireless power transfer (WPT) device as a function of 30 Gaussian uncertain parameters.

This paper is structured as follows: Section II briefly presents the mathematical background of the LS-SVM+GPR regression. Section III describes the PLS regression method as well as the sensitivity analysis derived from the method. Section IV presents the numerical results and compares the performance of the PLS surrogate with the ones provided by a 
LS-SVM+GPR surrogate model by considering the WPT testcase. Section V provides additional information on the features provided by each of the considered methods. Section VI concludes the paper.

\section{Probabilistic Model Combining LS-SVM and GPR}

Let us consider the problem of approximating a given set of $L$ training pairs $\left\{\left(\mathbf{x}_{i}, y_{i}\right)\right\}_{i=1}^{L}$ provided by a full computational model such that $y_{i}=\mathcal{M}\left(\mathbf{x}_{i}\right)$, as a function of its stochastic parameter $\mathbf{x}_{i} \in \mathcal{P}$ with $\mathcal{P} \subset \mathbb{R}^{d}$, in terms of the following GPR [13]:

$$
\mathcal{M}(\mathbf{x}) \approx \mu(\mathbf{x})+\varepsilon(\mathbf{x})
$$

where $\mu(\mathbf{x})$ is a deterministic function representing the mean estimated via a generic regression, also called trend and $\varepsilon(\mathbf{x}) \sim \mathcal{G P}\left(0, k\left(\mathbf{x}, \mathbf{x}^{\prime}\right)\right)$ is a Gaussian process (GP) with zero mean and covariance $k\left(\mathbf{x}, \mathbf{x}^{\prime}\right)$ approximating the deviation of the deterministic model from the actual function $\mathcal{M}$, for any configuration of the parameters $\mathbf{x}$. A GP can be considered as an extension of the concept of multivariate Gaussian distributions to infinite dimensionality which returns, for any arbitrary value of $\mathbf{x}_{*}$, a prediction of the mean value $\mu_{\mathbf{x}_{*}}$ and the variance $\sigma_{\mathbf{x}_{*}}^{2}$ of a normal distribution.

The covariance function $k\left(\mathbf{x}, \mathbf{x}^{\prime}\right)$ of the GP specifies the correlation between pairs of random variables under the assumption that $\mathcal{M}$ is smooth, this means that points with similar predictor values are expected to have close response values. The covariance function can be defined by several kernels, however, hereafter in this paper we will consider the Matern $3 / 2$ covariance function [13]:

$$
k\left(\mathbf{x}, \mathbf{x}^{\prime}\right)=\sigma_{f}^{2}\left(1+\frac{\sqrt{3}\left\|\mathbf{x}-\mathbf{x}^{\prime}\right\|}{\sigma_{l}}\right) \exp \left(\frac{-\sqrt{3}\left\|\mathbf{x}-\mathbf{x}^{\prime}\right\|}{\sigma_{l}}\right)
$$

where $\|\cdot\|$ represents the Euclidean distance and $\sigma_{f}$ and $\sigma_{l}$ are the hyper-parameters of the covariance estimated via an optimization procedure, e.g., by minimizing the negative log marginal likelihood of the GP.

Knowing the prior information provided by the training pairs $\left\{\left(\mathbf{x}_{i}, \tilde{y}_{i}\right)\right\}_{i=1}^{L}$, with $\tilde{y}_{i}=y_{i}-\mu\left(\mathbf{x}_{i}\right)$, for any new value of the input parameter $\mathbf{x}_{*} \in \mathcal{P}$ for $\mathbf{x}_{*} \neq \mathbf{x}_{i}$, the set of samples $\left\{\tilde{y}_{1}, \ldots, \tilde{y}_{L}, \tilde{y}_{*}\right\}$ follows an $L+1$-dimensional joint Gaussian distribution [17], which writes:

$$
\left[\begin{array}{c}
\tilde{\mathbf{y}} \\
\tilde{y}_{*}
\end{array}\right] \sim \mathcal{N}\left(\left[\begin{array}{l}
\mathbf{0} \\
0
\end{array}\right],\left[\begin{array}{cc}
\mathbf{K} & \mathbf{k}_{*}^{t} \\
\mathbf{k}_{*} & k_{* *}
\end{array}\right]\right),
$$

where $\tilde{\mathbf{y}}=\left[\tilde{y}_{1}, \ldots, \tilde{y}_{L}\right]^{t}, \mathbf{K} \in \mathbb{R}^{L \times L}$ is the correlation matrix in which each $i j$-element is given by $K_{i j}=k\left(\mathbf{x}_{i}, \mathbf{x}_{j}\right), \mathbf{k}_{*}=$ $\left[k\left(\mathbf{x}_{*}, \mathbf{x}_{1}\right), \ldots, k\left(\mathbf{x}_{*}, \mathbf{x}_{L}\right)\right] \in \mathbb{R}^{1 \times L}$ and $k_{* *}=k\left(\mathbf{x}_{*}, \mathbf{x}_{*}\right)$.

Therefore, for a generic configuration of the parameters $\mathbf{x}_{*} \in \mathcal{P}$, the probabilistic model in (1) provides as output a Gaussian distributed stochastic variable $M\left(\mathbf{x}_{*}\right) \sim$ $\mathcal{N}\left(\mu_{\mathbf{x}_{*}}, \sigma_{\mathbf{x}_{*}}^{2}\right)$ defined by the posterior mean $\mu_{\mathbf{x}_{*}}$ and variance $\sigma_{\mathbf{x}_{*}}^{2}$ :

$$
\mu_{\mathbf{x}_{*}}=\mu\left(\mathbf{x}_{*}\right)+\mathbf{k}_{*} \mathbf{K}^{-1} \tilde{\mathbf{y}}
$$

$$
\sigma_{\mathbf{x}_{*}}^{2}=k_{* *}-\mathbf{k}_{*} \mathbf{K}^{-1} \mathbf{k}_{*}^{t} .
$$

The prediction mean $\mu_{\mathbf{x}_{*}}$ is used instead of the deterministic model $\mu(\mathbf{x})$, whereas the variance $\sigma_{\mathbf{x}_{*}}^{2}$ gives a local error indicator about its precision. Different from the standard GP regression, hereafter in this work, we focus on the case of a GP regression with a fixed mean function [17].

In the proposed LS-SVM+GPR regression, we are assuming that the trend $\mu(\mathbf{x})$ of the GP in (1) is a known function previously estimated via a LS-SVM regression [8]. The LSSVM regression is considered since it allows building accurate and compact surrogate models of the response of a generic high-dimensional nonlinear function $\mathcal{M}$ starting from a limited number of training samples and writes:

$$
\mu(\mathbf{x})=M_{L S S V M}(\mathbf{x})=\sum_{i=1}^{L} \alpha_{i} K\left(\mathbf{x}_{i}, \mathbf{x}\right)+b
$$

where $\alpha_{i} \in \mathbb{R}$ are scalar coefficients, $K(\cdot, \cdot): \mathbb{R}^{d} \rightarrow \mathbb{R}$ is the kernel function and $b \in \mathbb{R}$ is the bias term.

It is important to remark that the kernel $K(\cdot, \cdot)$ in the above LS-SVM regression in the dual space is different from the covariance function $k(\cdot, \cdot)$ adopted by the GPR. In this work, the LS-SVMLab MATLAB Toolbox, version 1.8 [31] is used to build the LS-SVM regression model.

Summarizing, in the probabilistic model given in (1), the mean function $\mu(\mathbf{x})$ is built with the LS-SVM regression, whereas the model residuals $\varepsilon(\mathbf{x})$ are approximated by a GP regression. The resulting model not only approximates the system responses for any configuration of the input parameters, but it also provides an estimation of the prediction uncertainty.

\section{Partial Least Squares}

Partial Least Squares (PLS) [21] is a statistical method that enables to identify a linear relationship between input variables and the response variable. This technique relies on the projection of input variables onto a new space defined by new variables called principal components, which are linear combinations of the input variables. This method is well adapted when the input data set contains a small number of samples and a large number of input variables highly correlated to each other.

The principle of the PLS technique is based on the search of the best directions in the $\mathbf{X}$ space including the input variables, in order to explain the behavior of the response $\mathbf{y}$. First, the input matrix $\mathbf{X}$ of size $(N \times M)$, where $N$ and $M$ are the number of samples and input variables, respectively, and the response vector $\mathbf{y}$ are centered and reduced in order to avoid a bias of the input variables having large values and strong variations. Then, the first principal component $\mathbf{t}^{(1)}$ is computed by searching the best direction $\mathbf{u}^{(1)}$ maximizing the squared covariance between $\mathbf{t}^{(1)}=\mathbf{X} \mathbf{u}^{(1)}$ and $\mathbf{y}$ as:

$$
\mathbf{u}^{(1)}=\arg \max _{\mathbf{u}^{t} \mathbf{u}=1} \mathbf{u}^{t} \mathbf{X}^{t} \mathbf{y} \mathbf{y}^{t} \mathbf{X} \mathbf{u} .
$$

The optimization problem (6) is maximized when $\mathbf{u}^{(1)}$ is the eigenvector of the matrix $\mathbf{X}^{t} \mathbf{y} \mathbf{y}^{t} \mathbf{X}$ associated with the eigenvalue with the largest absolute value. The vector $\mathbf{u}^{(1)}$, called loading vector, corresponds to the $\mathbf{X}$ weights of the 
first component. Then, the residual matrix of $\mathbf{X}=\mathbf{X}^{(0)}$ and $\mathbf{y}=\mathbf{y}^{(0)}$, which are denoted by $\mathbf{X}^{(1)}$ and $\mathbf{y}^{(1)}$, are computed as:

$$
\begin{aligned}
\mathbf{X}^{(1)} & =\mathbf{X}^{(0)}-\mathbf{t}^{(1)} \mathbf{b}^{(1)}, \\
\mathbf{y}^{(1)} & =\mathbf{y}^{(0)}-\mathrm{w}_{1} \mathbf{t}^{(1)}
\end{aligned}
$$

with $\mathbf{b}^{(1)}$ representing a vector of size $(1 \times M)$ including the regression coefficients of the local regression of $\mathbf{X}$ onto the first principal component $\mathbf{t}^{(1)}$, and $\mathrm{w}_{1}$ is the regression coefficient of the local regression of $\mathbf{y}$ onto the first principal component $\mathbf{t}^{(1)}$. The equation (7) corresponds to the local regression of $\mathbf{X}$ and $\mathbf{y}$ onto the first principal component.

Then, PLS computes the second principal component, which is orthogonal to $\mathbf{t}^{(1)}$, replacing $\mathbf{X}$ by $\mathbf{X}^{(1)}$ and $\mathbf{y}$ by $\mathbf{y}^{(1)}$ so that the problem (6) is solved again. The same technique is used to compute the rest of the components with an iterative procedure. As an illustration, Fig. 1 represents the method in a three-dimensional example by considering two principal components. The number of principal components retained is denoted by $q$. In the rest of the paper, the number of principal components will be selected by retaining the one minimizing the root mean square error (RMSE) given by:

$$
R M S E=\frac{\sum_{i=1}^{N}\left(\hat{y}_{i}-y_{i}\right)^{2}}{N}
$$

where $N, y_{i}$ and $\hat{y}_{i}$ are the sample size, the real values and the predictions of the model, respectively.

The new coordinate system, defined by the principal components, corresponds to a rotation of the initial system defined by $\mathbf{x}_{1}, \ldots, \mathbf{x}_{M}$. The $r$-th component denoted $\mathbf{t}^{(r)}$, where $r=1, \ldots, q$, is given by:

$$
\mathbf{t}^{(r)}=\mathbf{X}^{(r-1)} \mathbf{u}^{(r)}=\mathbf{X} \mathbf{u}_{*}^{(r)} .
$$

The matrix $\mathbf{U}_{*}=\left[\mathbf{u}_{*}^{(1)}, \ldots, \mathbf{u}_{*}^{(q)}\right]$ is given by:

$$
\mathbf{U}_{*}=\mathbf{U}\left(\mathbf{B}^{t} \mathbf{U}\right)^{-1}
$$

with $\mathbf{U}=\left[\mathbf{u}^{(1)}, \ldots, \mathbf{u}^{(q)}\right]$ and $\mathbf{B}=\left[\mathbf{b}^{(1)^{t}}, \ldots, \mathbf{b}^{(q)^{t}}\right]$.

The vector $\mathbf{u}^{(r)}$ defines the principal direction in the initial space, maximizing the covariance of $\mathbf{X}^{(r-1)^{t}} \mathbf{y}^{(r-1)} \mathbf{y}^{(r-1)^{t}} \mathbf{X}^{(r-1)}$. When $q=M$, the matrix $\mathbf{U}_{*}=\left[\mathbf{u}_{*}^{(1)}, \ldots, \mathbf{u}_{*}^{(M)}\right]$ rotates the space defined by $\left(\mathbf{x}_{1}, \ldots, \mathbf{x}_{M}\right)$ to the new space defined by $\left(\mathbf{t}^{(1)}, \ldots, \mathbf{t}^{(M)}\right)$, oriented by the principal directions $\left(\mathbf{u}^{(1)}, \ldots, \mathbf{u}^{(M)}\right)$.

The variables importance projections (VIP) [23] in PLS is a variable selection method allowing to identify the importance of each input variables. It assumes that the column space of $\mathbf{X}$ has a subspace of dimension $q$ containing all relevant information for predicting the model response $\mathbf{y}$.

The VIP score for the input variable $j$ is defined as:

$$
V I P_{j}=\sqrt{\frac{M \cdot\left(\sum_{r=1}^{q} R^{2}\left(\mathbf{y}, \mathbf{t}^{(r)}\right) \cdot\left(\mathbf{u}^{(r j)} /\left\|\mathbf{u}^{(r j)}\right\|\right)^{2}\right)}{\sum_{r=1}^{q} R^{2}\left(\mathbf{y}, \mathbf{t}^{(r)}\right)}}
$$

where $M$ is the number of input variables, $\mathbf{u}^{(r j)}$ is the weight of the $j^{t h}$ variable in component $r$ and $R^{2}\left(\mathbf{y}, \mathbf{t}^{(r)}\right)$ is the percentage of $\mathbf{y}$ explained by the component $r$. The VIP value
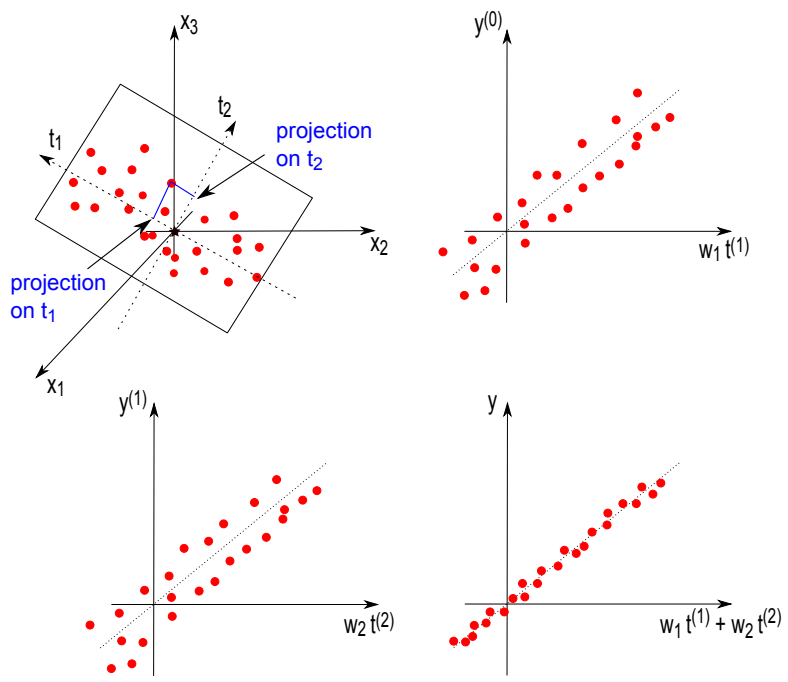

Fig. 1. Top left represents the construction of two principal components in the initial X space. Top right and Bottom left illustrate the prediction of $y^{(0)}$ and $y^{(1)}$, respectively. Bottom right shows the final prediction of the output $y$.

is a weighted sum of squares of the PLS weights $\left(\mathbf{u}^{(r j)}\right)$, which takes into account the explained variance of each PLS dimension. The input variables with a VIP score greater than one are generally considered to have a significant impact on the response $\mathbf{y}$ [23].

\section{Application Example: Wireless Power TRANSFER}

This Section investigates the feasibility and the strength of the GP+LS-SVM and the PLS regression techniques presented in Sec. II and III. Specifically, the above approaches are adopted to quantify the impact of fabrication tolerances and uncertainties on the maximal rectification efficiency, denoted $E_{f f}$, of the wireless power transfer (WPT) application given in Fig. 2 over [500, 1500] MHz frequency range (additional details on the considered test case are provided in [8], [29], [30]). For this purpose, the stochastic behavior of the efficiency has been analyzed by considering 30 Gaussian random variables centered at their nominal values and with a standard deviations of $5 \%$ of the nominal values, as given in Tab. I [8].

The LS-SVM+GPR and the PLS regressions are used hereafter to develop a probabilistic and deterministic surrogate model of the WPT efficiency in order to quantify the impact of uncertainties and reduce the computational cost with respect to MC simulation. A full-wave EM solver is used.

Both the LS-SVM+GPR and the PLS-based surrogate models are built by using the same set of training samples consisting of 200 realizations from Latin Hypercube sampling (LHS) [28]. As the PLS method is able to capture the behavior of an output with a small number of principal components, we construct a PLS surrogate model with a number of components varying from one to five. In order to evaluate the performance of each PLS model, a comparison with the brute force MC simulation with 10,000 realizations is carried out. Table II presents a comparison of each PLS model with respect to the 


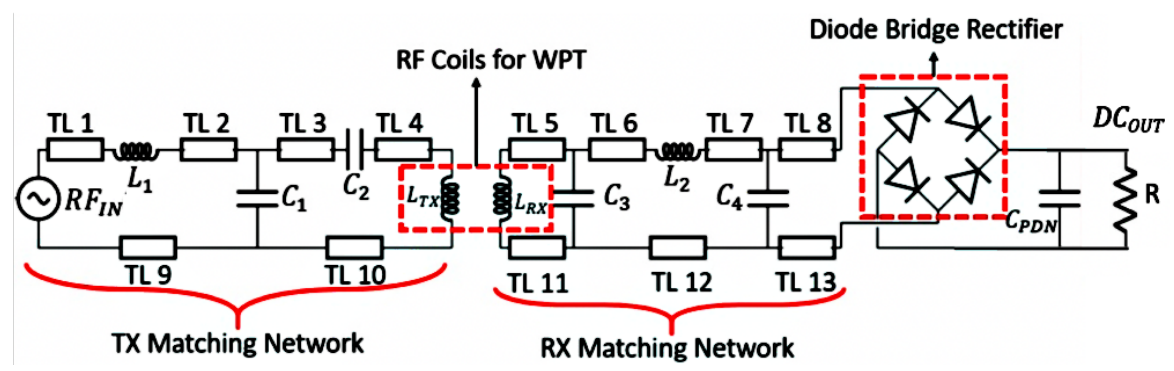

Fig. 2. Schematic of the WPT based power delivery architecture considered.

TABLE I

UNCERTAIN PARAMETERS CONSIDERED FOR THE WPT APPLICATION EXAMPLE.

\begin{tabular}{|c|c|c|c|c|}
\hline \multicolumn{2}{|c|}{ Gaussian Random Variables } & \multirow{2}{*}{$\begin{array}{l}\text { Unit } \\
\mathrm{mm}\end{array}$} & \multirow{2}{*}{$\begin{array}{c}\mu \\
2.46\end{array}$} & \multirow{2}{*}{$\frac{\sigma}{0.1230}$} \\
\hline Feeding Gap for TX coil & $g_{f, T X}$ & & & \\
\hline Feeding Gap for RX coil & $g_{f, R X}$ & $\mathrm{~mm}$ & 2.3 & 0.1150 \\
\hline Width \& Height of TX coil & $g_{\{x, y\}, T X}$ & $\mathrm{~mm}$ & 2.1 & 0.1050 \\
\hline Width \& Height of RX coil & $g_{\{x, y\}, R X}$ & $\mathrm{~mm}$ & 3 & 0.1500 \\
\hline GND Cut-Out Ratio TX & $\operatorname{slot}_{\{x, y\}, T X}$ & - & 1.19 & 0.0595 \\
\hline GND Cut-Out Ratio RX & $\operatorname{slot}_{\{x, y\}, R X}$ & - & 1.16 & 0.0580 \\
\hline Match Capacitor TX & $C_{1}$ & $\mathrm{pF}$ & 4.09 & 0.2045 \\
\hline Resonance Capacitor TX & $C_{2}$ & $\mathrm{pF}$ & 4.59 & 0.2295 \\
\hline Resonance Capacitor RX & $C_{3}$ & $\mathrm{pF}$ & 2.16 & 0.1080 \\
\hline Match Capacitor RX & $C_{4}$ & $\mathrm{pF}$ & 0.3 & 0.015 \\
\hline Match inductor TX & $L_{1}$ & $\mathrm{nH}$ & 1.25 & 0.0625 \\
\hline Match inductor RX & $L_{2}$ & $\mathrm{nH}$ & 7.82 & 0.3910 \\
\hline Line Width TX Coil & $l_{w, T X}$ & $\mathrm{~mm}$ & 1.89 & 0.0945 \\
\hline Line Width RX Coil & $l_{w, R X}$ & $\mathrm{~mm}$ & 0.71 & 0.0355 \\
\hline Width TL1 & $w_{T L 1}$ & $\mathrm{~mm}$ & 0.381 & 0.0191 \\
\hline Width TL2 & $w_{T L 2}$ & $\mathrm{~mm}$ & 0.407 & 0.0204 \\
\hline Width TL3 & $w_{T L 3}$ & $\mathrm{~mm}$ & 0.386 & 0.0193 \\
\hline Width TL4 & $w_{T L 4}$ & $\mathrm{~mm}$ & 0.528 & 0.0264 \\
\hline Width TL5 & $w_{T L 5}$ & $\mathrm{~mm}$ & 0.521 & 0.0191 \\
\hline Width TL6 & $w_{T L 6}$ & $\mathrm{~mm}$ & 0.397 & 0.0198 \\
\hline Width TL7 & $w_{T L 7}$ & $\mathrm{~mm}$ & 0.607 & 0.0303 \\
\hline Width TL8 & $w_{T L 8}$ & $\mathrm{~mm}$ & 0.520 & 0.0260 \\
\hline Length TL1 & $l_{T L 1}$ & $\mathrm{~mm}$ & 1.656 & 0.0828 \\
\hline Length TL2 & $l_{T L 2}$ & $\mathrm{~mm}$ & 1.424 & 0.0712 \\
\hline Length TL3 & $l_{T L 3}$ & $\mathrm{~mm}$ & 0.723 & 0.0362 \\
\hline Length TL4 & $l_{T L 4}$ & $\mathrm{~mm}$ & 2.153 & 0.0828 \\
\hline Length TL5 & $l_{T L 5}$ & $\mathrm{~mm}$ & 0.612 & 0.1076 \\
\hline Length TL6 & $l_{T L 6}$ & $\mathrm{~mm}$ & 1.666 & 0.0833 \\
\hline Length TL7 & $l_{T L 7}$ & $\mathrm{~mm}$ & 0.502 & 0.0251 \\
\hline Length TL8 & $l_{T L 8}$ & $\mathrm{~mm}$ & 0.555 & 0.0278 \\
\hline
\end{tabular}

number of components by computing the root mean square error (RMSE) over the 10,000 realizations, the mean value $\hat{\mu}$ and the standard deviation $\hat{\sigma}$ of the efficiency. We observe that the PLS model with one principal component provides a RMSE equal to 0.547 . Then, the PLS model, from two to five principal components, provides a RMSE of 0.535 and 0.536. It is worth noting that the PLS surrogate model with one component achieved a quite low RMSE, i.e. 0.547, which is very close to the lowest RMSE obtained by PLS with two principal components, i.e. 0.535. In the following, this last PLS surrogate model is selected as it is the most accurate. A similar comparison has been carried out for the LS-SVM+GPR surrogate model. For this specific model the RMSE is 0.488 , which is comparable with the one provided by the PLS with two components.

By comparison with several surrogate models used in [8] (see Tab. I), we notice that both the PLS surrogate model built-up using 200 LHS simulations is a bit less accurate than the LS-SVM surrogate model with a RBF kernel and more accurate than the sparse PC, which have shown their efficiency in complex problems. This highlights the capability of the PLS surrogate model to capture the main trend of a complex response based on a significant reduction of the dimensionality of the problem, i.e., from 30 initial variables to two principal components. This feature of the advocated method is very interesting and will be exploited in the following Section. On the other hand, the accuracy of the LS-SVM+GPR is the same of the one obtained by the LS-SVM surrogate model with RBF kernel.

As an illustration, Fig. 3 provides the scattering plots comparing the predictions of the LS-SVM+GPR and the PLS regression model with two principal components against the MC simulation with 10,000 realizations. We see a good agreement between the estimated efficiency by the proposed LSSVM+GP and PLS surrogate models compared with the one obtained from the results of the MC simulation, since the realizations are quite close to the dashed line representing a perfect surrogate model. The above qualitative comment on the model accuracy is also confirmed by the correlation coefficients which take the value of 0.704 for the LS-SVM+GPR model and 0.628 for the PLS model, thus confirming the capability of the proposed techniques to deal with complex problem with a limited set of training samples (i.e., 30 variables with only 200 samples). It is worth pointing out that the low values of the WPT efficiency, where the efficiency estimated by MC simulation is below $73 \%$, are not well predicted by the models 
TABLE II

COMPARISON AMONG THE PERFORMANCES OF THE PLS, SPARSE PC, LS-SVM (RBF) AND LS-SVM (RBF)+GPR-BASED MODELS OF THE WPT EFFICIENCY BUILT WITH 200 LHS SAMPLES W.R.T THE RESULTS OF A MC SIMULATION WITH 10,000 SAMPLES. THE NUMBERS IN BOLD FONT HIGHLIGHT THE RMSE OF THE MOST ACCURATE MODELS.

\begin{tabular}{|c|c|c|c|c|c|c|}
\hline Method & $\begin{array}{c}\text { Numberx } \\
\text { of Comp. }\end{array}$ & RMSE & $\hat{\mu}$ & $\hat{\sigma}$ & $t_{\text {model }}$ & $t_{\text {cost }}$ \\
\hline \hline MC & - & - & 74.19 & 0.68 & - & 11 days \\
\hline \hline \multirow{3}{*}{ PLS } & 1 & 0.547 & 74.20 & 0.45 & $5 \mathrm{~h}^{*}$ & $<1 \mathrm{~s}$ \\
\cline { 2 - 7 } & 2 & 0.535 & 74.20 & 0.50 & $5 \mathrm{~h}^{*}$ & $<1 \mathrm{~s}$ \\
\cline { 2 - 7 } & 3 & 0.536 & 74.20 & 0.51 & $5 \mathrm{~h}^{*}$ & $<1 \mathrm{~s}$ \\
\cline { 2 - 8 } & 4 & 0.536 & 74.20 & 0.51 & $5 \mathrm{~h}^{*}$ & $<1 \mathrm{~s}$ \\
\cline { 2 - 8 } & 5 & 0.536 & 74.20 & 0.51 & $5 \mathrm{~h}^{*}$ & $<1 \mathrm{~s}$ \\
\hline \hline LS-SVM(RBF)+GPR & - & $\mathbf{0 . 4 8 8}$ & 74.25 & 0.68 & $5 \mathrm{~h}^{*}$ & $<1 \mathrm{~s}$ \\
\hline \hline LS-SVM(RBF) [8] & - & $\mathbf{0 . 4 8 8}$ & 74.23 & 0.54 & $5 \mathrm{~h}^{*}$ & $<1 \mathrm{~s}$ \\
\hline \hline Sparse PC (Order 3) [8] & - & 0.565 & 74.25 & 0.52 & $5 \mathrm{~h}^{*}$ & $<1 \mathrm{~s}$ \\
\hline
\end{tabular}

200 LHS training simulations required $5 \mathrm{~h}$.
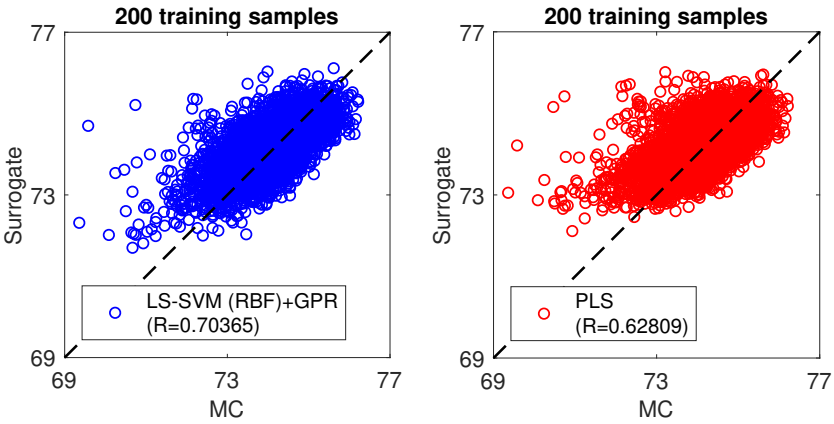

Fig. 3. Scatter plot of the WPT efficiency computed by the PLS surrogate model (red circles), the mean value of the LS-SVM (RBF)+GPR surrogate model (blue circles) vs. the actual numerical model with 10,000 MC realizations. The dashed line illustrates the perfect agreement between the models.

due to the strong non-linearity and discontinuities of the output in that region.

As the variability of the output is of interest, Fig. 4 shows the PDFs estimated by the LS-SVM+GP and PLS surrogate models and by $\mathrm{MC}$ simulation from 10,000 realizations. It can be observed that the LS-SVM+GP and PLS surrogate models capture well the main variability of the efficiency provided by MC simulation, although some discrepancies appear between the left tails of the probability distribution. Regarding the computational cost, 10,000 MC simulations required about 11 days while the PLS surrogate and the LS-SVM+GPR model needed less than $1 \mathrm{~s}$ to perform the same predictions. This comparison does not consider the computational cost required to generate the training data set (200 simulations), which took about $5 \mathrm{~h}$ as shown in Table II.

\section{Method Strengths}

The results provided in Sec. IV show that both proposed techniques allow providing accurate surrogate models for the considered test case by using a limited set of training samples. This section aims at highlighting the advantages and differences between the two modeling techniques.

\section{A. LS-SVM+GPR Probabilistic Model}

The LS-SVM+GPR probabilistic model presented in Sec. II is extremely useful and powerful, since it provides the user

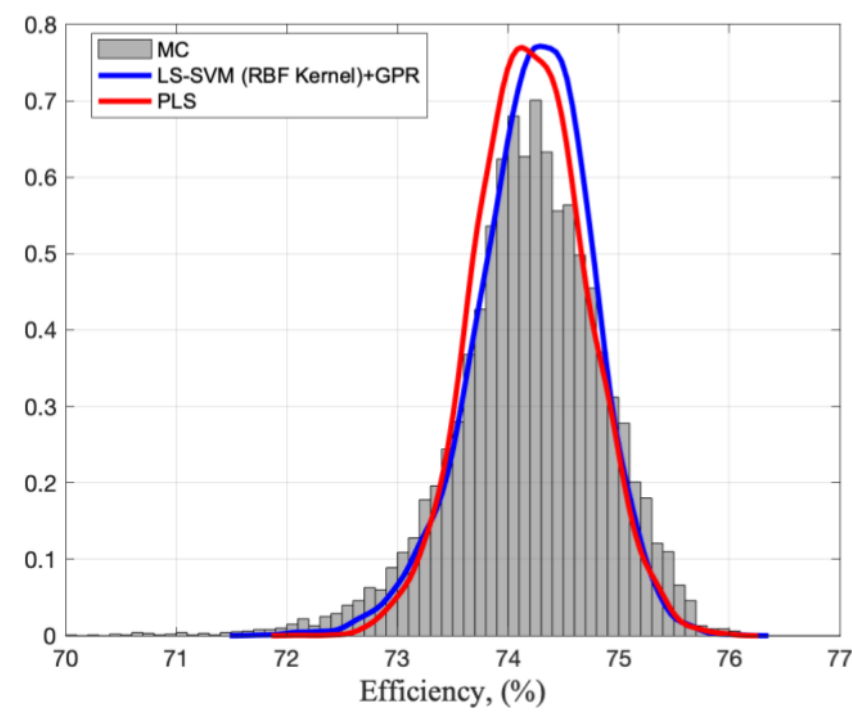

Fig. 4. PDFs of the WPT efficiency estimated by the PLS surrogate model (solid blue curve), the mean value of the LS-SVM (RBF)+GP surrogate model (solid red curve) and by the MC simulation (gray histogram) from 10,000 realizations.

with additional information on the model reliability. Indeed, for any configuration of the stochastic input variables $\mathbf{x}_{*}$, the proposed probabilistic model provides the output as a stochastic variable with a Gaussian PDF which writes:

$$
\mathcal{M}\left(\mathbf{x}_{*}\right) \sim N\left(\mu_{\mathbf{x}_{*}}, \sigma_{\mathbf{x}_{*}}^{2}\right)=\mathcal{M}_{L S-S V M+G P R}\left(\mathbf{x}_{*}\right)
$$

where $\mu_{\mathbf{x}_{*}}$ and $\sigma_{\mathbf{x}_{*}}^{2}$ are computed according to (4a) and (4b), respectively.

This means that each prediction obtained with the proposed probabilistic model is a Gaussian variable. The above description of the model output in terms of a PDF allows providing a probabilistic interpretation of the model uncertainty such as the model error, in which the prediction obtained with the model is a Gaussian variable. According to this, Fig. 5 provides a comparison between the PDF of the WPT efficiency computed from the results of a 10,000 MC simulations with the PDFs obtained by considering, for each configuration of the $\mathrm{MC}$ samples, 100 different realizations of the corresponding output $N\left(\mu_{\mathbf{x}_{*}}, \sigma_{\mathbf{x}_{*}}^{2}\right)$ in (12). The results show that the statistical information provided by the proposed probabilistic model allows providing a more conservative and reliable estimation of the actual statistical behavior of the efficiency, especially in tails of the distribution [14].

Moreover, the resulting probabilistic interpretation of the model realization allows estimating the confidence bounds on the model prediction for a given confidence interval (CI) of $100(1-\alpha) \%$ as follows:

$$
\left(\mu_{\mathbf{x}_{*}}-z_{1-\frac{\alpha}{2}} \sigma_{\mathbf{x}_{*}}\right) \leq \mathcal{M}\left(\mathbf{x}_{*}\right) \leq\left(\mu_{\mathbf{x}_{*}}+z_{1-\frac{\alpha}{2}} \sigma_{\mathbf{x}_{*}}\right),
$$

where $z$ denotes the inverse of the Gaussian cumulative distribution function evaluated at $1-\frac{\alpha}{2}$.

For the considered application example, the $99 \%$ CIs predicted by the proposed LS-SVM+GPR model allow providing a conservative estimation of the actual values of the WPT 


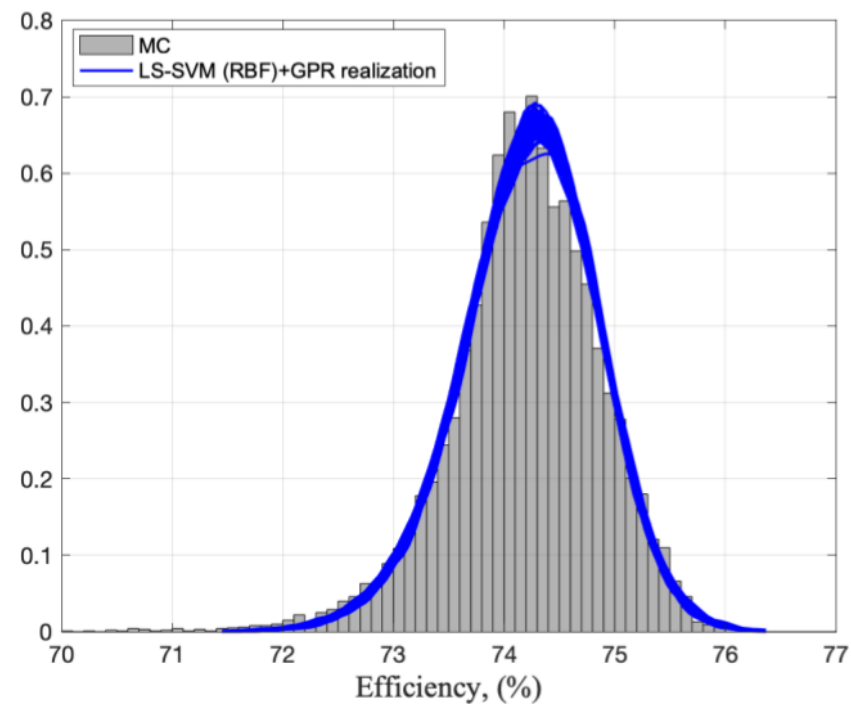

Fig. 5. Comparison between the PDFs of the WPT efficiency calculated from the results of $10,000 \mathrm{MC}$ simulations of the full-computational model (gray histogram) and the PDFs obtained by considering, for each configuration of the MC samples, 100 different realizations predicted by the proposed LSSVM+GPR probabilistic model (blue lines).

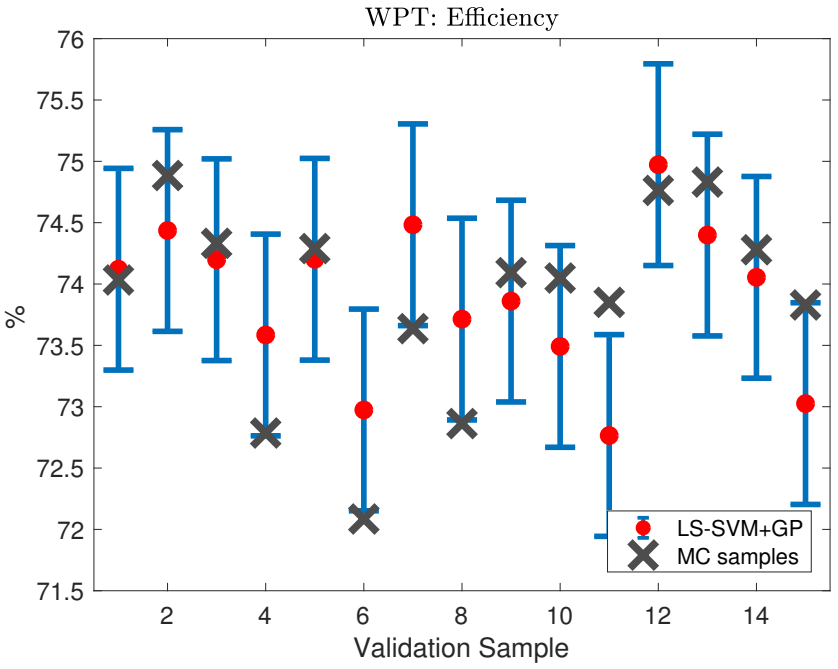

Fig. 6. Comparison between the results of 15 simulations of the fullcomputational model (black crosses) randomly chosen among the 10,000 MC samples with the corresponding mean value (red dots) and 99\% CI (horizontal blue bars) estimated via the LS-SVM+GPR probabilistic model.

efficiency for 91,280 cases out of 10,000 validation samples (i.e., $91.3 \%$ of the overall cases). For the sake of illustration, Fig. 6 shows the CIs predicted by the model for 15 validation samples randomly selected among the 10,000 samples used in the MC simulation. According to the results, the proposed model allows to accurately predict the model uncertainty, since most of the validation samples (black crosses) fall within the CIs predicted by the proposed model (blue bars).

\section{B. PLS \& Sensitivity Analysis}

Besides the estimation of the variability of the WPT efficiency, the PLS model allows to derive a sensitivity analysis of the output. Figure 7 presents a histogram of the VIP scores, which estimate the importance of each input variables in the projection used in the PLS model. From Figure 7, we observe that, out of 30 input random variables, eight variables have a significant impact, defined by a VIP score larger than or equal to 1 (dashed line) as suggested by [23], on the variability of the efficiency $E_{f f}$. In particular, the most important parameters are related to the transmitter and receiver coils, i.e., $g_{\{x, y\}, T X}$ and $g_{\{x, y\}, R X}, g_{f, R X}, \operatorname{slot}_{\{x, y\}, R X}$, respectively, as well as some components such as $L_{2}, C_{2}, C_{3}$ and $C_{4}$. The rest of the parameters, including the ones associated to the geometry of transmission lines (i.e., widths and lengths) have a negligible impact on the variability of the WPT efficiency.

The PLS surrogate also allows to analyze the correlation of such parameters on the WPT efficiency for the first two components (i.e., $t_{1}$ and $t_{2}$ ) via the correlation circle in Fig. 8. This correlation can be obtained by looking at the weight of each variables over the two components. More specifically, Fig. 8 shows that the projection of the output of interest (i.e. WPT efficiency $E_{f f}$ ) on the first component $t_{1}$ (which is the most important by construction) is equal to about 0.75 . We also notice that the correlation between the group of input variables composed of $L_{2}, C_{4}$, slot $\{x, y\}, R X, C_{3}, g_{\{x, y\}, R X}$ and the first component $t_{1}$ is about 0.5 . Since those variables (input and output variables) are positively correlated with the first component $t_{1}$, and relatively close to each other, this means that a higher value of these input variables will increase the output variable (i.e., the WPT efficiency $E_{f f}$ ). The rest of the input variables are not significantly correlated with the WPT efficiency $E_{f f}$ as their projection over the first component $t_{1}$ is quite low.

To demonstrate the validity of this information, for the input variables $L_{2}, C_{4}$, slot $\{x, y\}, R X, C_{3}, g_{\{x, y\}, R X}$, we increased the corresponding mean values provided in Tab. I of $2 \sigma$ (i.e., $\tilde{\mu}=\mu+2 \sigma$ ) while choosing a standard deviation of $\tilde{\sigma}=\sigma / 10$ in order to not exceed the upper bound of the input variables of the initial configuration. A new MC simulation with 10,000 samples has been run providing the new PDF (green bins) in Fig. 9. This illustration compares the PDF obtained with the optimized configuration of the system parameters (green histogram) with their initial configuration (grey histogram). The mean value and the standard deviation of the optimized and initial configurations are given by $\mu_{o p t}=74.89$ and $\sigma_{o p t}=0.74$, and $\mu_{i n i t}=74.19$ and $\sigma_{\text {init }}=0.68$, respectively, confirming that controlling a small group of input parameters can significantly improve the performance of the system.

\section{CONCLUSIONS}

This paper focuses on the application of the LS-SVM+GPR and the PLS technique to the development of surrogate models, with a very low computational cost compared to $\mathrm{MC}$ simulation, for the uncertainty quantification in high-dimensional space. The obtained models allowed to efficiently estimate the model output in a complex problem characterized by 30 uncertain input variables. The results of a 10,000 MC samples obtained with the full-computational model have been considered as reference results. The strength of the proposed technique along with their accuracy and computational cost are carefully discussed. From the results, the proposed modeling 


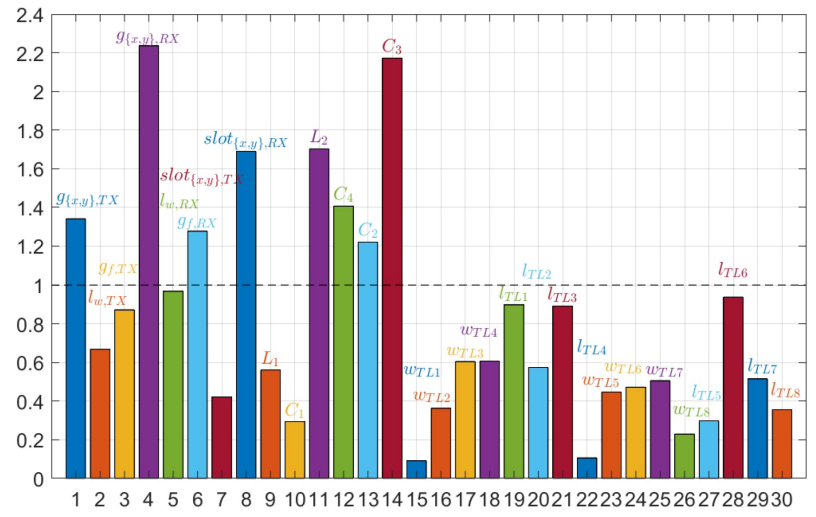

Fig. 7. Importance of the input parameters according to their impact on the behavior of the output.

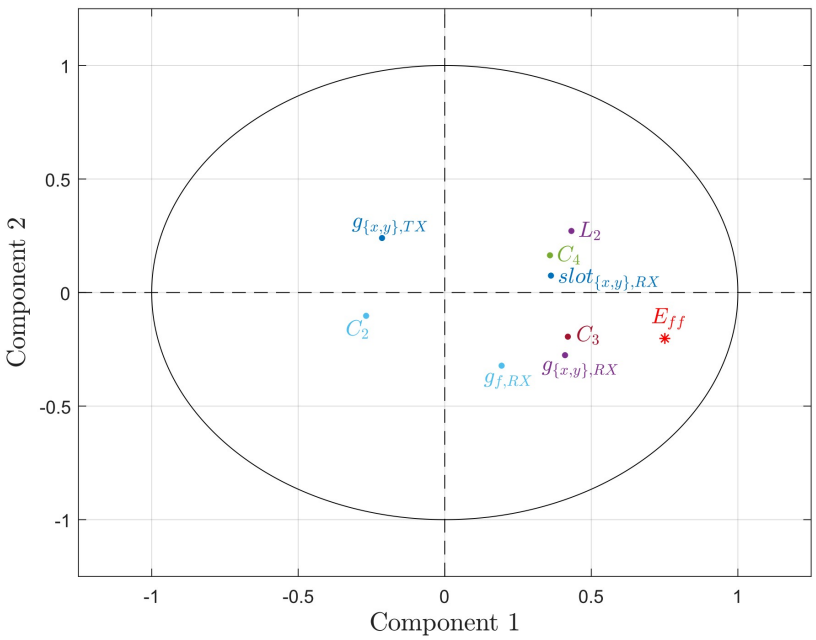

Fig. 8. Circle of correlation representing the projection of the input variables and the output variable (WPT efficiency $E_{f f}$ ) onto the plane defined by the first two PLS components. It shows a strong positive correlation between the input variables $L_{2}, C_{4}, \operatorname{slot}_{\{x, y\}, R X}, C_{3}, g_{\{x, y\}, R X}$ and the output variable $E_{f f}$ since their projections are in the same direction and at a significant distance from the origin.

schemes can be considered as valuable and viable solutions for the UQ of the output of complex systems in high-dimensional parameter space.

\section{REFERENCES}

[1] D. Xiu, and G. E. Karniadakis, "The Wiener-Askey polynomial chaos for stochastic differential equations," SIAM journal on scientific computing, vol. 24, no 2, pp. 619-644, 2002.

[2] I. S. Stievano, P. Manfredi and F. G. Canavero, "Stochastic Analysis of Multiconductor Cables and Interconnects," IEEE Trans. Electromagn. Compat., vol. 53, no. 2, pp. 501-507, May 2011.

[3] A. Kaintura, T. Dhaene, and D. Spina, "Review of polynomial chaosbased methods for uncertainty quantification in modern integrated circuits," Electronics, vol. 7, no. 3, p. 30:1-21, Feb. 2018.

[4] Z. Zhang, T. Weng, and L. Daniel, "Big-data tensor recovery for high-dimensional uncertainty quantification of process variations," IEEE Trans. Compon. Packag. Manuf. Technol., vol. 7, no. 5, pp. 687-697, May 2017.

[5] G. Blatman and B. Sudret, "Adaptive sparse polynomial chaos expansion based on least angle regression," Journal of Computational Physics, vol. 230 , no 6 , pp. $2345-2367,2011$.

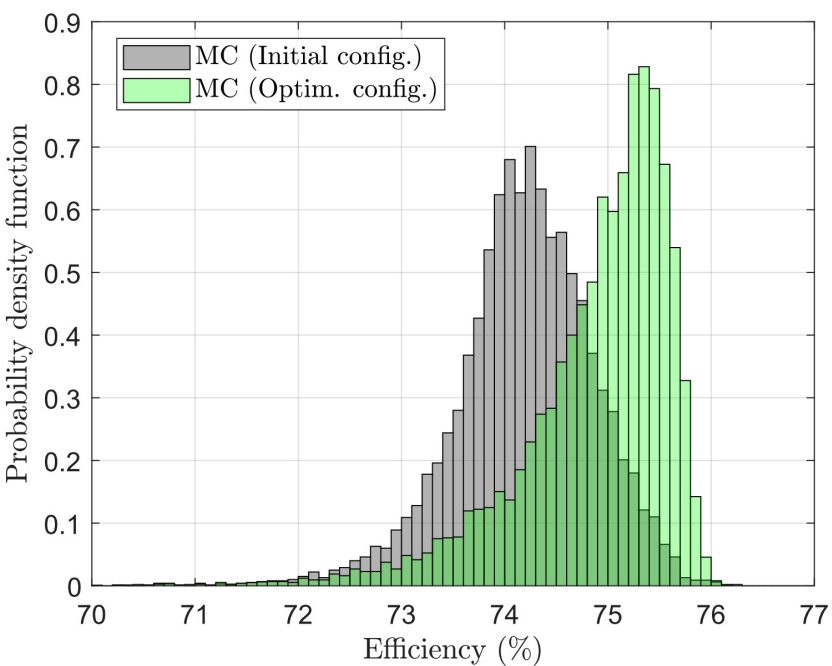

Fig. 9. PDF of the efficiency of the wireless power transfer improved (green) by increasing the nominal values of the input variables $L_{2}, C_{4}, \operatorname{slot}_{\{x, y\}}, R X$, $C_{3}, g_{\{x, y\}, R X}$ by $2 \sigma$, where $\sigma$ is given Table I, according to the circle of correlation given in Fig. 8.

[6] M. Ahadi and S. Roy, "Sparse linear regression (SPLINER) approach for efficient multidimensional uncertainty quantification of high-speed circuits," IEEE Trans. Comput.-Aided Des. Integr. Circuits Syst., vol. 35, no. 10 , pp. $1640-1652$, Oct. 2016.

[7] M. Larbi, I. S. Stievano, F. G. Canavero and P. Besnier, "Variability Impact of Many Design Parameters: The Case of a Realistic Electronic Link," IEEE Trans. Electromagn. Compat., vol. 60, no. 1, pp. 34-41, Feb. 2018.

[8] R. Trinchero, M. Larbi, H. M. Torun, F. G. Canavero and M. Swaminathan,, "Machine Learning and Uncertainty Quantification for Surrogate Models of Integrated Devices With a Large Number of Parameters," IEEE Access, vol. 7, no. 1, pp. 4056-4066, 2019.

[9] R. Trinchero, P. Manfredi, I. S. Stievano and F. G. Canavero, "Machine Learning for the Performance Assessment of High-Speed Links", IEEE Trans. Electromagn. Compat., vol. 60, no. 6, pp. 1627-1634, Dec. 2018.

[10] R. Trinchero and F. G. Canavero, "Design of Passive Equalizer for Space Wire Links via Support Vector Machine," in Proc. of the International Symposium on Electromagnetic Compatibility (EMC EUROPE), Amsterdam, 2018, pp. 53-56.

[11] R. Trinchero, I. S. Stievano and F.G. Canavero, "Black-Box Modeling of the Maximum Currents Induced in Harnesses During Automotive Radiated Immunity Tests", IEEE Trans. Electromagn. Compat., vol. 62 , no. 2, pp. 627-630, April 2020.

[12] R. Trinchero, F. G. Canavero, "Machine Learning for the Design of a Distribution Network for High-Speed Signals", in Proc. of the 2019 International Conference on Electromagnetics in Advanced Applications (ICEAA), Granada, Spain, 2019, pp. 1038-1041.

[13] C. E. Rasmussen and C. K. I. Williams, Gaussian Processes for Machine Learning, MIT Press. Cambridge, Massachusetts, 2006.

[14] T. Houret, P. Besnier, S. Vauchamp and P. Pouliguen, "Controlled Stratification Based on Kriging Surrogate Model: An Algorithm for Determining Extreme Quantiles in Electromagnetic Compatibility Risk Analysis," IEEE Access, vol. 8, pp. 3837-3847, 2020.

[15] R. Trinchero, M. Larbi, M. Swaminathan and F. G. Canavero, "Statistical Analysis of the Efficiency of an Integrated Voltage Regulator by means of a Machine Learning Model Coupled with Kriging Regression," in Proc. IEEE 23rd Workshop on Signal and Power Integrity (SPI), Chambry, France, 2019, pp. 1-4.

[16] R. Trinchero and F. G. Canavero, "Probabilistic Model of the Effect of a Ground Discontinuity on the Transmission of a Microstrip Interconnect," 2019 International Symposium on Electromagnetic Compatibility (EMC EUROPE), Barcelona, Spain, 2019, pp. 908-911.

[17] R. Trinchero and F. G. Canavero, "Combining LS-SVM and GP Regression for the Uncertainty Quantification of the EMI of Power Converters Affected by Several Uncertain Parameters," IEEE Trans. Electromagn. Compat. (Early Access). DOI: 10.1109/TEMC.2019.2962899.

[18] A. K. Prasad and S. Roy, "Accurate Reduced Dimensional Polyno- 
mial Chaos for Efficient Uncertainty Quantification of Microwave/RF Networks," IEEE Transactions on Microwave Theory and Techniques, vol. 65, no. 10, pp. 3697-3708, Oct. 2017.

[19] J. Tang, F. Ni, F. Ponci and A. Monti, "Dimension-Adaptive Sparse Grid Interpolation for Uncertainty Quantification in Modern Power Systems: Probabilistic Power Flow," IEEE Trans. Power Syst., vol. 31, no. 2, pp. 907-919, March 2016.

[20] C. Cui and Z. Zhang, "High-Dimensional Uncertainty Quantification of Electronic and Photonic IC with Non-Gaussian Correlated Process Variations," IEEE Tran. Comput.-Aided Design Integr. Circuits Syst. (Early Access). DOI: 10.1109/TCAD.2019.2925340.

[21] L. E. Frank, and J. H. Friedman, "A statistical view of some chemometrics regression tools", Technometrics, Taylor \& Francis, 1993, vol. 35, no. 2, pp. 109-135.

[22] S. Wold and K. Esbensen and P. Geladi, "Principal component analysis", Chemometrics and intelligent laboratory systems, Elsevier, 1987, vol. 2, no. $1-3$, pp. 37-52.

[23] I. G. Chong, and C. H. Jun, "Performance of some variable selection methods when multicollinearity is present", Chemometrics and intelligent laboratory systems, Elsevier, 2005, vol. 78, no. 1-2, pp. 103-112.

[24] H. M. Torun, M. Swaminathan, A. Kavungal Davis and M. L. F. Bellaredj, "A Global Bayesian Optimization Algorithm and Its Application to Integrated System Design," in IEEE Trans. on Very Large Scale Integration (VLSI) Systems, vol. 26, no. 4, pp. 792-802, April 2018.

[25] S. Mueller, K. Z. Ahmed, A. Singh, A. K. Davis, S. Mukhopadyay, M. Swaminathan, Y. Mano, Y. Wang, J. Wong, S. Bharathi, H. Fathi Moghadam and D. Draper, "Design of high efficiency integrated voltage regulators with embedded magnetic core inductors," in Proc. IEEE 66th Electronic Components and Technology Conf., May 2016, pp. 566-573.

[26] Ansys HFSS ver. 2015.2, http://www.ansys.com

[27] F. Pedregosa, G. Varoquaux, A. Gramfort, V. Michel,B. Thirion, O. Grisel, M. Blondel, P. Prettenhofer, R. Weiss, V. Dubourg, J. Vanderplas, A. Passos, D. Cournapeau, M. Brucher, M. Perrot, E. Duchesnay, "Scikit-learn: Machine Learning in Python," Journal of Machine Learning Research, vol. 12, pp. 2825-2830, 2011.

[28] M. McKay, R. Beckman and W. Conover, "A comparison of three methods for selecting values of input variables in the analysis of output from a computer code", Technometrics, vol. 42, no. 1, pp. 55-61, 2000.

[29] R. Jay and S. Palermo, "Resonant coupling analysis for a two-coil wireless power transfer system," in Proc. IEEE Dallas Circuits and Systems Conference (DCAS), Oct 2014, pp. 1-4.

[30] H. M. Torun, C. Pardue, M. L. F. Belleradj, A. K. Davis and M. Swaminathan, "Machine Learning Driven Advanced Packaging and Miniaturization of IoT for Wireless Power Transfer Solutions," in Proc. IEEE 68th Electronic Components and Technology Conference (ECTC), San Diego, CA, 2018, pp. 2374-2381.

[31] LS-SVMlab, version 1.8; Department of Electrical Engineering (ESAT), Katholieke Universiteit Leuven: Leuven, Belgium, 2011. Available online: http://www.esat.kuleuven.be/sista/lssvmlab/.

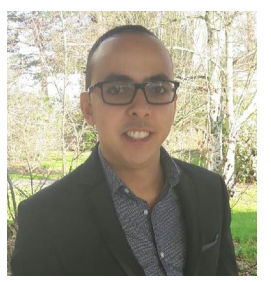

Mourad Larbi received the M.S. degree in applied statistics from the University of Nice SophiaAntipolis, Nice, France, in 2011, and the Ph.D. degree in electronics and telecommunications from the Institute of Electronics and Telecommunications of Rennes (IETR), Rennes, France, in 2016. He then worked, from 2016 to 2017, as a Post-Doctoral Researcher with the Electromagnetic Compatibility Group, Department of Electronics and Telecommunications, Politecnico di Torino, Turin, Italy. He is currently working as a Post-Doctoral Researcher at the 3D Systems Packaging Research Center (PRC), School of Electrical and Computer Engineering, Georgia Institute of technology, GA, USA. His current research interests concern behavioral modeling and the risk analysis of signal propagation on interconnects in high-dimensional uncertainty quantification problems. Dr. Larbi received the Richard B. Schulz Best EMC Transactions Paper Award - Honorable Mention in 2016 and won the Best Paper Award at the IEEE International Symposium and Exhibition on Electromagnetic Compatibility (EMC Europe) in 2017.

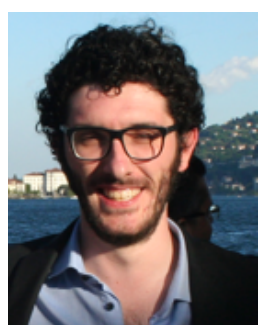

Riccardo Trinchero (M'16) received the M.Sc. and the Ph.D. degrees in Electronics and Communication Engineering from Politecnico di Torino, Torino, Italy, in 2011 and 2015, respectively. He is currently an Assistant Professor within the EMC Group with the Department of Electronics and Telecommunications at the Politecnico di Torino. His research interests include the analysis of linear time-varying systems, modeling and simulation of switching converters and statistical simulation of circuits and systems.

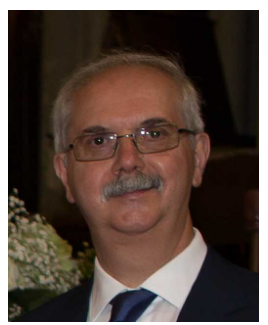

Flavio G. Canavero (SM'99-F'07) received his electronic engineering degree from Politecnico (Technical University) of Torino, Italy, and the $\mathrm{PhD}$ degree from the Georgia Institute of Technology, Atlanta, USA, in 1986. Currently he is a Professor of Circuit Theory with the Department of Electronics and Telecommunications, Politecnico di Torino, where he serves also as the Director of the Doctoral School. He is an IEEE Fellow. He has been the Editor-in-Chief of IEEE Transactions on Electromagnetic Compatibility, V.P. for Communication Services of the EMC Society and Chair of URSI Commission E. He received several Industry and IEEE Awards, including the prestigious Richard R. Stoddard Award for Outstanding Performance, which is the EMC Society?s highest technical award, and the Honored Member Award of EMC Society. His research interests include signal integrity and EMC design issues, interconnect modeling, black-box characterization of digital integrated circuits, EMI and statistics in EMC.

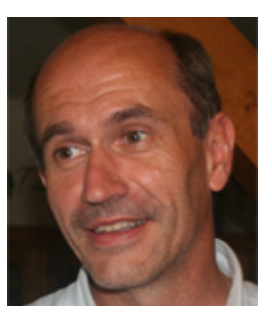

Philippe Besnier (M'04, SM'10) received the diplôme d'ingénieur degree from Ecole Universitaire d'Ingénieurs de Lille (EUDIL), Lille, France, in 1990 and the Ph. D. degree in electronics from the university of Lille in 1993. Following a one-year period at ONERA, Meudon as an assistant scientist in the EMC division, he was with the laboratory of radio-propagation and electronics (LRPE), University of Lille, as a researcher (chargé de recherche) at the Centre National de la Recherche Scientifique (CNRS) from 1994 to 1997. From 1997 to 2002, Philippe Besnier was the director of Centre d'Etudes et de Recherches en Protection Electromagnétique (CERPEM): a non-for-profit organization for research, expertise and training in EMC and related activities, based in Laval, France. He co-founded TEKCEM in 1998 a small business company specialized in turnkey systems for EMC measurements. Back to CNRS in 2002, he has been since then with the Institute of Electronics and Telecommunications of Rennes (IETR). Philippe Besnier was appointed as CNRS senior researcher (directeur de recherche au CNRS) in 2013. He was co-head of the "antennas and microwave devices" research department of IETR between 2012 and 2016. He headed the WAVES (electromagnetic waves in complex media) team during the first semester of 2017. Since July 2017, he is now a deputy director of IETR. His research activities are mainly dedicated to interference analysis on cable harnesses (including electromagnetic topology), theory and application of reverberation chambers, near-field probing and uncertainty quantification in EMC modeling. 


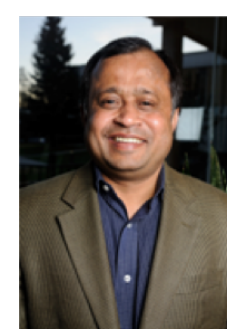

Madhavan Swaminathan (M'95-SM'98-F'06) received the M.S. and Ph.D. degrees in electrical engineering from Syracuse University in 1989 and 1991, respectively. He was with IBM, where he was involved in packaging for supercomputers. He was the Joseph M. Pettit Professor in electronics with the School of ECE and the Deputy Director of the NSF Microsystems Packaging Research Center, Georgia Tech. He is currently the John Pippin Chair Professor in microsystems packaging and electromagnetics with the School of Electrical and Computer Engineering (ECE) and the Director of the Center for CoDesign of Chip, Packages, System, Georgia Institute of Technology (Georgia Tech), Atlanta, GA, USA. He is also the Founder and the Co-Founder of two start-up companies, E-System Design and Jacket Micro Devices. He is the author of over 450 refereed technical publications, holds 29 patents, and the primary author and a co-editor of three books. He is the Founder of the IEEE Conference Electrical Design of Advanced Packaging and Systems, a premier conference sponsored by the CPMT Society on Signal Integrity in the Asian region. He has served as the Distinguished Lecturer for the IEEE EMC Society. 